\title{
HER-2-positive gastric cancer accompanied by liver metastasis: a case report of a rare complete response and literature review
}

\author{
Hongmei Yong ${ }^{1 \Delta}$, Yeping Du ${ }^{2 \Delta}$,Wei Wang ${ }^{1}$, Huichang Shi ${ }^{1}$, Ronghui Cheng ${ }^{1}$, Xuan Jiang ${ }^{1}$, Xueyi Zhou ${ }^{1 *}$ \\ ${ }^{1}$ Department of Oncology, Huai'an Hospital Affiliated to Xuzhou Medical College and the Second Huai'an People's Hospital, \\ Huai'an, Jiangsu, 223002, China; \\ ${ }^{2}$ Department of Intensive Care Unit, Huai'an Hospital Affiliated to Xuzhou Medical College and the Second Huai'an People's Hospital, \\ Huai'an, Jiangsu, 223002, China.
}

\begin{abstract}
The prognosis of advanced gastric cancer is poor, and the prognosis of late-stage gastric cancer with the expression of human epidermal growth factor receptor 2 (HER-2) is worse. Personalized therapy is based on the specific situation of each patient to determine the best treatment plan. We diagnosed a 36-year-old patient with a HER-2-producing adenocarcinoma of the gastric cardia accompanied by liver metastasis. Sixteen cycles of chemotherapy were consecutively administered to the patient, starting with a three-drug combination, followed by a two-drug combination, and finally a single drug as a chemotherapy regimen. During these treatments, the molecular targeted drug trastuzumab was continuously administered. In the end, the patient experienced a complete response (CR). In clinical practice, advanced gastric cancer is not well controlled in certain patients, due to inadequate first-line chemotherapy and its side-effects. If a patient enjoys a strong performance status (PS), we emphasize the importance of molecular-targeted drugs combined with prolonged administration of chemotherapy.
\end{abstract}

Keywords: HER-2, gastric cancer, liver metastasis, chemotherapy

\section{INTRODUCTION}

The median overall survival (OS) time of patients with metastatic gastric cancer is only about 4 months ${ }^{[1]}$. Personalized therapy offers treatment regimens based on molecular diagnosis, including targeted therapy and individualized chemotherapy. Human epidermal growth factor receptor 2 (HER2 ) is a transmembrane tyrosine kinase (TK) receptor related to tumor cell differentiation, proliferation, ap-

\footnotetext{
*Correspondence to: Dr. Xueyi Zhou, Department of Oncology, Huai'an Hospital Affiliated to Xuzhou Medical College and the Second Huai'an People's Hospital, Huai'an, Jiangsu, 223002, China. TEL: 0086-517-80871779. E-mail: cjshazjj@163.com.

Conflict of Interest: The authors declare that they have no conflict of interest.

${ }^{\Delta}$ These authors contributed equally to this work.
}

optosis, migration, and adhesion. It is overexpressed in around $15 \%$ to $20 \%$ of patients with gastroesophageal junction and primary gastric cancers ${ }^{[2,3]}$. A phase III gastric cancer clinical trial (ToGA trial) showed that trastuzumab (Herceptin), as a humanized monoclonal antibody against HER-2, could effectively prolong OS and progression-free survival (PFS) in HER-2-positive advanced gastric carcinoma ${ }^{[4]}$. Many studies have suggested that many malignant tumors including gastric cancer may be more aggressive due to high expression levels of HER-2 expres$\operatorname{sion}^{[5-7]}$. Another study showed trastuzumab combined with chemotherapy to be effective for HER-2 positive advanced gastric cancer patients ${ }^{[8]}$. However, it is extremely rare to achieve a complete response in the first-line of treatment for metastatic advanced gastric cancer. Our study describes a patient with HER-2 ${ }^{+}$ specific gastric cancer accompanied by liver metasta- 
sis who was treated with personalized therapy based targeted therapy and many cycles of individualized chemotherapy. Written informed consent was obtained from the patient.

\section{Case report}

A 36-year-old female patient was admitted to the Huai'an Hospital Affiliated to Xuzhou Medical College (Huai'an, Jiangsu, China) on August 01, 2016 for the sensation of poststernal obstruction after eating. Endoscopic examination revealed a new growth about $40 \mathrm{~cm}$ from the incisors, surface erosion and blockage of the lumen (Fig.1A). The pathological biopsy indicated that it was an adenocarcinoma of the gastric cardia. A computed tomography (CT) scan showed the liver to be occupied in multiple positions and accompanied by intrahepatic bile duct expansion (Fig.2A). Further liver biopsy pathology showed hepatic adenocarcinoma and possible metastasis. The liver tumors were immunohistochemically positive for CK19, CK7, villin, and carcino-embryonic antigen (CEA), and negative for CK20 and hepatocytes. These observations were consistent with liver metastasis of gastric adenocarcinoma. The patient was found to be positive for HER-2 expression (HER-2/CEPI7=5.42) by FISH.

Due to the high level of HER-2 expression, we developed an individualized molecular targeted therapy combined with chemotherapy for the patient. Treatment with the molecular targeted drug Herceptin lasted a total of ten months $(8 \mathrm{mg} / \mathrm{kg} \mathrm{dl}, 6 \mathrm{mg} / \mathrm{kg}$ $\mathrm{q} 3 \mathrm{w})$. Triple combination chemotherapy of docetaxel, cisplatin, and 5-fluorouracil (DCF) is the standard treatment regimen for unresectable advanced gastric cancer in the National Comprehensive Cancer Network (NCCN) guide. Several modified schedules $(\mathrm{mDCFs})$ are widely used in clinical practice duo to their relatively low levels of acute toxicity and are considered viable as first-line chemotherapies for advanced gastric cancer ${ }^{[9-12]}$. Treatment with six courses of mDCF chemotherapy was initiated (docetaxel 75 $\mathrm{mg} / \mathrm{m}^{2}$ on day 1 , oxaliplatin $100 \mathrm{mg} / \mathrm{m}^{2}$ on day 1 and S-1 $80 \mathrm{mg} / \mathrm{m}^{2}$ on days $1-14$ every 3 weeks). The first evaluation of the efficacy of the therapeutic schedule took place after the patient had undergone two courses of mDCF-regimen chemotherapy. The multiple metastatic liver lesions were found to be significantly smaller by CT scan (Fig. 2 B). However, the patient's carbohydrate antigen (CA) 724 serum level (normal range, $0-7.6 \mathrm{kU} / \mathrm{L}$ ) had increased significantly from 2.01 to $13.97 \mathrm{kU} / \mathrm{L}$. As the treatment progressed, the CA724 serum level peaked at $39.96 \mathrm{U} / \mathrm{mL}$ after three cycles of chemotherapy.
We evaluated the efficacy by CT in accordance with the evaluation criteria of RECIST 1.1 every two cycles. After six courses of mDCF chemotherapy, a follow-up CT scan showed that the multiple metastatic liver lesions had grown still smaller. Upon evaluation, the effect was considered to be a partial response (PR) (Fig. 2C). However, the patient's CA724 serum levels $(16.88 \mathrm{kU} / \mathrm{L})$ did not decrease into the normal range.

In order to further control the disease, we administered the patient with personalized chemotherapy using anoxaliplatin $+\mathrm{S}-1$ regimen (oxaliplatin $100 \mathrm{mg} / \mathrm{m}^{2}$ on day 1 and $\mathrm{S}-180 \mathrm{mg} / \mathrm{m}^{2}$ on days $1-14$, every 4 weeks). After two courses of chemotherapy with anoxaliplatin + S-1 regimen, the patient's CA724 serum level $(4.1 \mathrm{kU} / \mathrm{L})$ had returned to the normal range. However, the CT examination indicated that there was still a large number of occupying lesions in the liver after four courses of chemotherapy with the oxaliplatin $+\mathrm{S}-1$ regimen, and the maximum diameter of these lesions was less than $1.0 \mathrm{~cm}$. Because of the patient's good PS, we subsequently administered further personalized chemotherapy in the form of single-
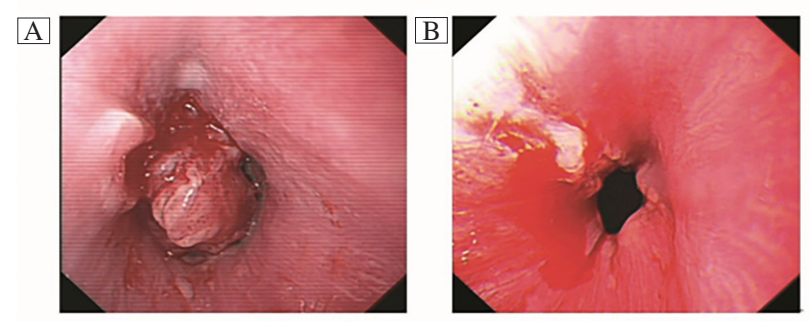

Fig. 1 Representational image of gastroscopy. (A) The large occupying lesion obstructed the lumen with superficial erosion on August 01, 2016. (B) These lesions in the gastric cardia had disappeared on August 15, 2017.

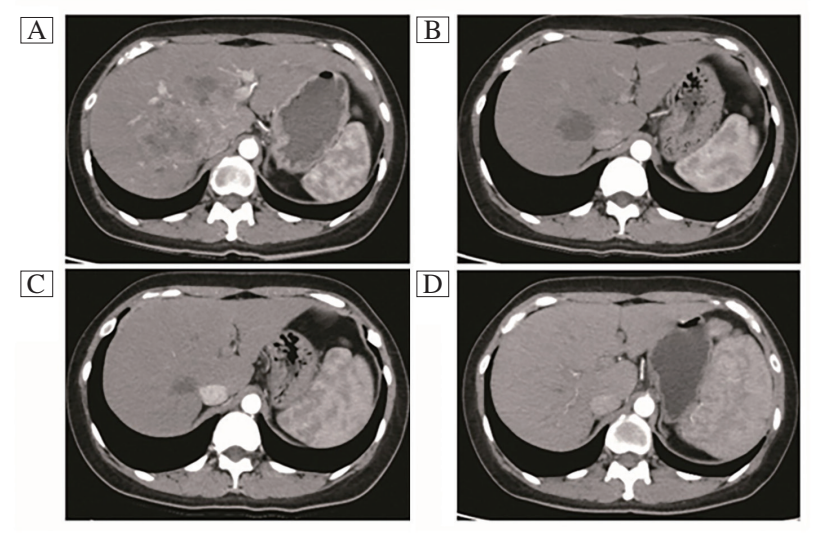

Fig. 2 Abdominal computed tomography scans revealing. (A) Multiple and massive liver metastases prior to therapy; (B) Partial remission of the metastatic liver lesions after two cycles of Doc + Oxa+ S-1 chemotherapy; (C) Partial remission of the metastatic liver lesions after six cycles of Doc + Oxa+ S-1 chemotherapy; (D) Liver lesions all disappeared following 5 cycles of S-1 chemotherapy. Doc, docetaxel; Oxa, oxaliplatin. 
drug S-1 chemotherapy for six cycles $\left(\mathrm{S}-180 \mathrm{mg} / \mathrm{m}^{2}\right.$ on days $1-5,8-12,15-19$, every 4 weeks). A sixth evaluation took place after five cycles of S-1 chemotherapy on August 15, 2017. Using a CT scan, the metastatic liver lesions were found to have responded completely (Fig. 2D). Endoscopic examination performed at roughly the same time showed that all lesions in the gastric cardia had completely disappeared (Fig. 1B).

At no point during treatment was there any visible abnormality in tumor markers, such as the alphafetal protein, iron protein, CA125, or CA199, or any change in the level of CA724 expression (as shown in Fig. 3). The patient's PS remained between 0 and 1. There was no significant gastrointestinal reaction, such as anorexia, nausea, or vomiting. The most serious hematology toxicity was that the leukocytes achieved degree III bone marrow suppression, which improved

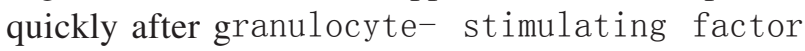
(GSF) treatment. The lowest level of platelets was degree II bone marrow suppression, which improved slowly without any special treatment. As of this writing, the patient has experienced no disease progression for more than 4 months and maintains a relatively active lifestyle.

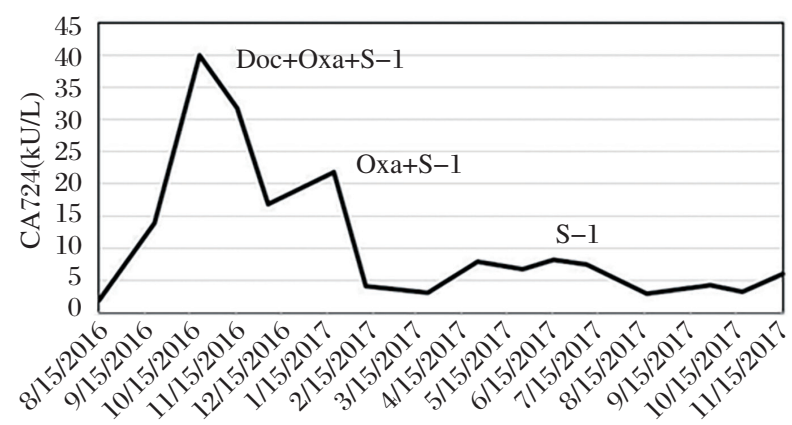

Fig. 3 Analysis of CA724 serum levels. Marker serum level gradually increased before three cycles of Doc + Oxa+ S-1 chemotherapy. The CA724 serum level then decreased, but it did not return to normal until the second round of Oxa+ S-1 chemotherapy. It then remained within the normal range for almost nine months. CA724, carbohydrate antigen 724; Doc, docetaxel; Oxa, oxaliplatin.

\section{DISCUSSION}

Despite recent advances in genetic diagnosis and new therapeutic strategies, patients with advanced unresectable gastric cancer still have a poor survival rate $^{[4]}$. The ToGA study indicated that patients with advanced gastric cancer for HER-2 positive expression had a median OS of 13.8 months when treated with trastuzumab in conjunction with cisplatin plus fluorouracil or capecitabine. However, patients in the control group, who received chemotherapy alone, only had a median OS of 11.1 months. Because the ToGA results are published, the program of trastuzumab combined with chemotherapy was recommended as a first-line treatment for patients with advanced unresectable gastric cancer positive for HER-2. Then a recent study came to similar conclusions ${ }^{[8]}$. However, few patients achieved a CR when the two treatments were combined.

The present case reports a CR, whose diagnosis was extensively supported by gastroscopy and CT imaging, which occurred in a patient with advanced gastric cancer accompanied by liver metastasis treated with trastuzumab and sixteen cycles of chemotherapy. Early use of trastuzumab and individualized chemotherapy results in a lack of clinical benefits to patients.

HER-2 was found to have been overexpressed in various carcinomas, but it has been widely studied in breast and gastric carcinomas ${ }^{[13-15]}$. HER-2, as an oncogene product, is an important predictive factor, mainly because it promotes the growth of tumor cells and metastasis of the whole body ${ }^{[16]}$. Other molecules such as epidermal growth factor receptor (EGFR), vascular endothelial growth factor (VEGF), c-Met and hepatocyte growth factor (HGF) have also been investigated for their significant roles in promoting the growth of gastric cancer and as potential targets for treatment ${ }^{[17,18]}$. For this reason, early use of a targeted drug is particularly helpful for the prognosis of advanced gastric cancer.

There have been no previous reports covering the transition of the therapeutic model from three chemotherapeutic drugs to two drugs and then one drug in a patient with advanced gastric cancer. The efficacy of treatment in this patient was still PR after six cycles of three-drug combination chemotherapy and four cycles of two-drug combination chemotherapy. Gastroscopy and $\mathrm{CT}$ scans indicated that the disease reached CR in the fifth course of treatment of single drug S-1 chemotherapy. To further control the disease, we continued to maintain the $\mathrm{S}-1$ regimen chemotherapy for one full course. For this reason, we believe that it is very important for patients with advanced gastric cancer to have a full dose and course of chemotherapy, even if CR is not reached after six cycles of first-line chemotherapy.

In this patient, the CA724 serum level was normal before chemotherapy, and its expression gradually increased as the $\mathrm{mDCF}$ chemotherapy regimen continued. When the third round of mDCF chemotherapy was completed, the CA724 serum level reached its peak value, but CT evaluation showed the curative effect to be PR. Subsequently, the serum levels 
of CA724 gradually returned its normal range. The likely cause of this phenomenon is that the patient had a massive tumor load in the stomach and liver before chemotherapy, and CA724 was released from a large number of necrotic tumor cells after chemotherapy. For this reason, the clinical practice further proves that it is not possible to judge the efficacy of chemotherapy based on the tumor markers, so it is necessary to evaluate the efficacy of the tumor chemotherapy by $\mathrm{CT}$ according to the RECIST 1.1 standard criteria version.

The patient had a good PS both before and after chemotherapy, which was the basis for the continued use of individualized chemotherapy. For this reason, it is worth learning the targeted therapy model combined with individualized chemotherapy for otherwise healthy patients, so that more HER-2 positive patients with advanced gastric cancer can benefit more from first-line treatment.

\section{Acknowledgements}

We thank LetPub (www.letpub.com) for its linguistic assistance during the preparation of this manuscript.

\section{References}

[1] Yang D, Hendifar A, Lenz C, et al. Survival of metastatic gastric cancer: Significance of age, sex and race/ethnicity. J Gastrointest Oncol, 2011, 2(2):77-84.

[2] Begnami MD, Fukuda E, Fregnani JHTG, et al. Prognostic implications of altered human epidermal growth factor receptorl(HERs) in gastric carcinomas: HER2 and HER3 are predictors of poor outcome. J Clin Oncol, 2011, 29(22):3030-6.

[3] Kurokawa Y, Matsuura N, Kimura Y, et al. Multicenter large-scale study of prognostic impact of HER2 expression in patients with resectable gastric cancer. Gastric Cancer, 2015, 18(4):691-7.

[4] Bang YJ, Van CE, Feyereislova A, et al. Trastuzumab in combination with chemotherapy versus chemotherapy alone for treatment of HER2-positive advanced gastric or gastro-oesophageal junction cancer (ToGA): a phase 3, open-label, randomised controlled trial. Lancet, 2010, 376(9742): 687-97.

[5] Hu B, Hajj NE, Sittler S, et al. Gastric cancer: Classification, histology and application of molecular pathology. $J$ Gastrointest Oncol, 2012, 3(3):251-61.

[6] Rajagopal I, Niveditha SR, Sahadev R, et al. HER 2 ex- pression in gastric and gastro-esophageal junction (GEJ) Adenocarcinomas. J Clin Diagn Res, 2015, 9(3):EC06-10.

[7] Yun S, Koh J, Nam SK, et al. Clinical significance of overexpression of NRG1 and its receptors, HER3 and HER4, in gastric cancer patients. Gastric Cancer, 2017, DOI: 10.1007/s10120-017-0732-7.

[8] Namikawa T, Munekage E, Munekage M, et al. Evaluation of a trastuzumab-containing treatment regimen for patients with unresectable advanced or recurrent gastric cancer. Mol Clin Oncol, 2016, 5(1):74-8.

[9] Petrelli F, Tomasello G, Ghidini M, et al. Modified schedules of DCF chemotherapy for advanced gastric cancer: a systematic review of efficacy and toxicity. Anticancer Drugs, 2017, 28(2):133-41.

[10] Shah MA, Janjigian YY, Stoller R, et al. Randomized multicenter phase ii study of modified docetaxel, cisplatin, and fluorouracil (DCF) versus DCF plus growth factor support in patients with metastatic gastric adenocarcinoma: A study of the US Gastric Cancer Consortium. J Clin Oncol, 2015, 33(33):3874-9.

[11] Chen Y, Yang J, Cai X, et al. The efficacy and toxicity of modified docetaxel, cisplatin and 5-fluorouracil combination therapy for 27 patients with advanced stage gastric adenocarcinoma. The Chinese-German Journal of Clinical Oncology, 2010, 9(7):396-400.

[12] Chi Y, Ren JH, Yang L, et al. Phase II clinical study on the modified DCF regimen for treatment of advanced gastric carcinoma. Chin Med J, 2011, 124(19):29973002.

[13] Gravalos C, Jimeno A. HER2 in gastric cancer: a new prognostic factor and a novel therapeutic target. Ann Oncol, 2008, 19(9):1523-9.

[14] Wang SC, Hung MC. HER2 overexpression and cancer targeting. Semin Oncol,2001, 28(16):115-24.

[15] Koeppen HKW, Wright BD, Burt AD, et al. Overexpression of HER2/ neu in solid tumours: an immunohistochemical survey. Histopathology, 2001, 38(2):96-104.

[16] Jeung J, Patel R, Vila L, et al. Quantitation of HER2/neu. Expression in primary gastresophageal adenocarcinomas using conventional light microscopy and quantitative image analysis. Arch Pathol Lab Med, 2012, 136(6):610-7.

[17] Schinzari G, Cassano A, Orlandi A, et al. Targeted therapy in advanced gastric carcinoma: the future is beginning. Curr Med Chem, 2014, 21(8):1026-38.

[18] Yalcin S, Yildiz Y and Sokmensuer C. Evaluation of cMet, HGF, and HER-2 expressions in gastric carcinoma and their association with other clinicopathological factors. Onco Targets Ther, 2016, 9:5809-17.

(Received 10 February 2018, Revised 03 March 2018, Accepted 06 March 2018) 